\title{
Experimental Studies on the Effect of Antenna Orientations to the Performance of OFDM-based System
}

\author{
J. Muslimin, A.L. Asnawi, A.F. Ismail, A.Z. Jusoh, N.A. Malek, H.A.M. Ramli \\ Department of Electrical and Computer Engineering, International Islamic University, Malaysia
}

\begin{tabular}{l}
\hline \hline Article Info \\
\hline Article history: \\
Received Mar 11, 2018 \\
Revised May 29, 2018 \\
Accepted Jun 7, 2018 \\
\hline
\end{tabular}

Keyword:

Antenna orientations

Experimental

OFDM

SDR

USRP

\begin{abstract}
Software-defined radio (SDR) is an emerging and promising high reconfigurable platform for rapid prototyping inreal environment applications. It offers both flexibility and low cost to facilitate the development process of agile communication system, such as Orthogonal Frequency Division Multiplexing (OFDM). Other than modulation and transmission technique like OFDM, antenna orientations play a significant importance in wireless communication. The availabililty of SDR platform like USRP has enabled the empirical evaluation of antenna orientation to the system performance. The performance has been evaluated in terms of throughput and packet error rate. The findings show the antenna orientation affect the system performance significantly.
\end{abstract}

Copyright @ 2018 Institute of Advanced Engineering and Science. All rights reserved.

\section{Corresponding Author:}

\section{A.L. Asnawi}

Department of Electrical and Computer Engineering, International Islamic University, Jalan Gombak, 53100 Kuala Lumpur, Malaysia.

Email: jusnainimuslimin@gmail.com

\section{INTRODUCTION}

Research in the field of wireless communication is vast and dynamic due to the endless demand of the end-user and the scarcity of the wireless spectrum [1]. Wireless communication plays a great importance in human daily life. It applies in almost every aspects of human activities, ranging from social, commercial, weather forecast, military, civilian fields, to domestic appliances. The exponential growth of wireless demand has urged for technique and mechanism to improve the existing communication system. As such, a widely deployed technique such as OFDM has gained a lot of attention among researches as it offers both efficient spectrum utilization and robust high data rate transmission in frequency selective fading. The studies on OFDM are extensive and the literatures are abundance [2]-[10]. For instance, [11] studied the implementation of OFDM in both WiMAX and LTE for cognitive radio system, while [12]-[14] studied the OFDM usage in optical system. OFDM common application in cellular network is depicted in [15] where the writer focused on 4G cellular network. In [16]-[18], OFDM were studied in the field of RADAR. However, as compared to the simulation analyses, experimental analyses were less explored.

Meanwhile, the concept of software-defined radio (SDR) offers a breakthrough for expensive, time consuming and effort demanding of building an RF applications.It changed the hardware problem of RF application into software problem which grants a flexibility to change the system functionality by means of software alone, which is ideal for rapid development of communication prototype. However, although the SDR hardware like Universal Software Radio Peripheral (USRP) is emerging, the platform technology is not mature yet. Thus, room for exploration is widely opened. Therefore, this study has emulated an OFDM-based system on the SDR platform of USRP with GNU Radio and has used the setup to evaluate the effect of antenna orientations to the system performance. The system performance has been empirically evaluated in terms of percentage of packet error rate and throughput. 


\section{RESEARCH METHOD}

This part describes the implemented OFDM system and experimentations setup used in this study.

\subsection{System design and setup}

In this study, the OFDM-based transceiver has been realized in programming scripts on GNU Radio software environment while utilizing a pair of USRPs as the hardware platform. The source code of both Transmitter and Receiver has been implemented in hierarchical manner with 'transmit path' and 'receive_path' are at the top of the hierarchy as shown in Figure 1. Both 'transmit_path' and 'receive_path' provide interface for transmitter and receiver respectively in which the system configurations and functionalities were provided by abstraction from source codes at the lower hierarchical.



Figure 1. Architecture of implemented OFDM-based system

Meanwhile, the setup of the testbed consists of GNU Radio, a pair of USRP, workstation, and antenna. The system software processing was implemented in GNU Radio in which was installed on Ubuntu 14.04 operating system. A pair of USRPs N210 with mounted CBX-40MHz was deployed as the hardware platform of the system. The USRPs were connected to the workstation via Ethernet gigabit cable. The workstation was a set of i7 $2.7 \mathrm{GHz}$ octa-core PC and i7 $2.4 \mathrm{GHz}$ octa-core desktop which were assigned as transmitter and receiver respectively as depicted in Figure 2.

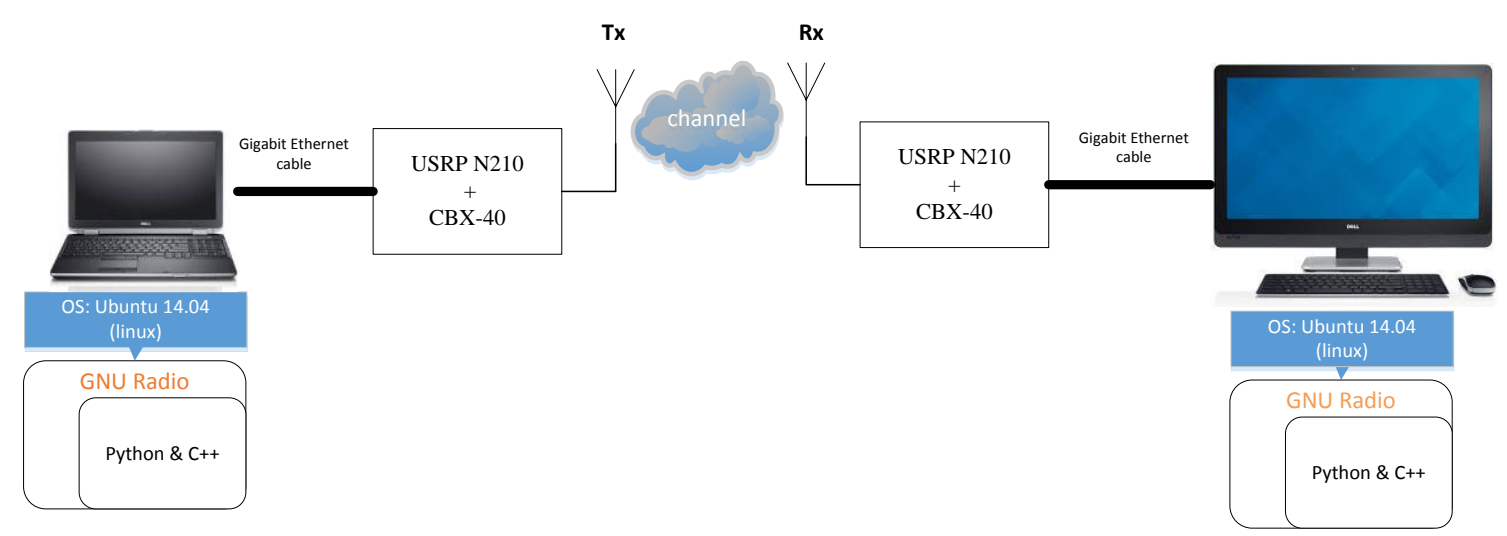

Figure 2. Experimental setup

\subsection{Antenna measurement}

Two types of antennas were used in this study named omnidirectional monopole antenna and bidirectional patch antenna. Prior to the experimentations, the Return Loss (S11) of these antennas was measured using Agilent Vector Network Analyzer (VNA) as shown in Figure 3. While antennas that been 
used were expected to operate at LTE and Wifi frequencies band for patch bi-directional and monopole omnidirectional antennas respectively, the return loss experienced at the experimented frequencies are shown in Figure 3.
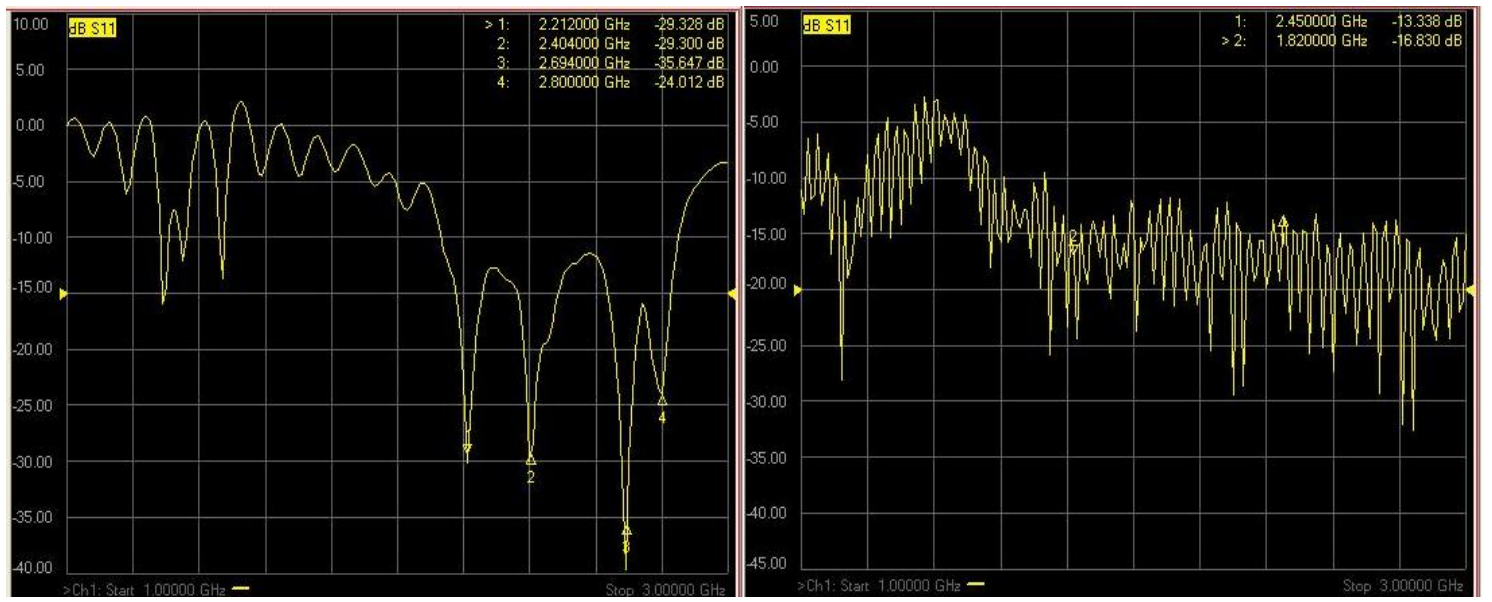

Figure 3. Measured S11 of patch bi-directional antenna (left) and monopole omni-directional antenna (right)

From Figure 3, patch antenna shows the lowest return loss at $2.694 \mathrm{GHz}$ which is $-35.647 \mathrm{~dB}$. Meawhile, no significant difference of noise level shown by omnidirectional between the measured frequencies. However, since omnidirectional shows higher return loss than patch antenna, this study was anticipating the patch antenna at $2.6 \mathrm{GHz}$ will give better throughput and error rate than the omnidirectional antenna at $2.4 \mathrm{GHz}$.

\subsection{Evaluated antenna orientations}

The effect of polarization or orientation of monopole and patch antenna with omnidirectional and bidirectional radiation pattern respectively to the performance of OFDM-based communication system were varied and evaluated. For monopole antenna, the antenna has been installed to the USRP in either vertical or horizontal position as shown in Figure 4. The combination of antenna orientation at transmitter and receiver gives either parallel or perpendicular configuration which labeled as Vertical - Vertical (V-V), Vertical - Horizontal (V-H), Horizontal - Vertical (H-V), and Horizontal - Horizontal (H-H). The order of these combinations refers to the orientation of the antenna on the transmitter followed by the receiver.

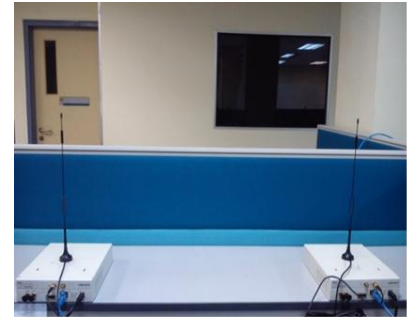

a) $V(T x)-V(R x)$

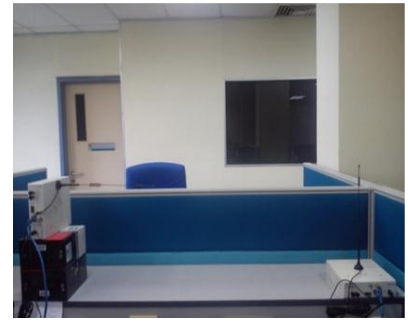

c) $H(T x)-V(R x)$

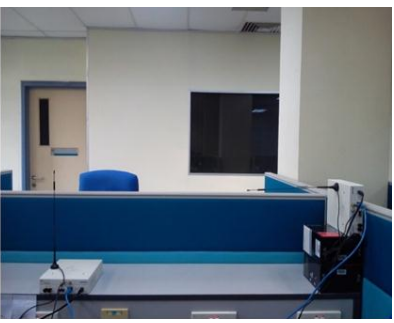

b) $\mathrm{V}(\mathrm{Tx})-\mathrm{H}(\mathrm{rx})$

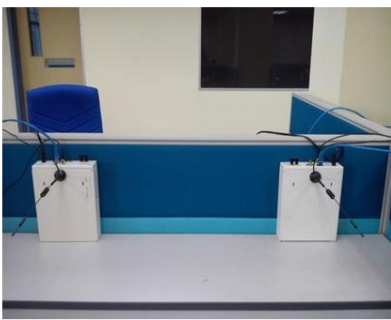

d) $H(T x)-H(R x)$

Figure 4. Orientations of monopole omnidirectional antenna 
Likewise, the effect of polarization or orientation of antenna with bi-directional radiation pattern was evaluated by positioning the antenna to be either facing each other or facing outward and labeled as 'in-in', 'in-out', 'out-in', and 'out-out'as shown in Figure 5.

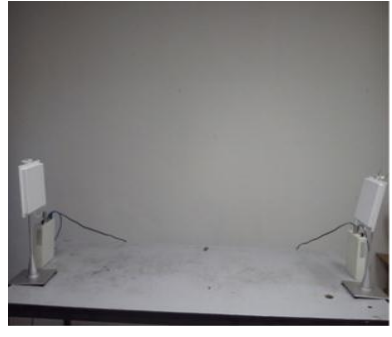

a) $\mathrm{Tx}-\mathrm{Rx}$ facing in

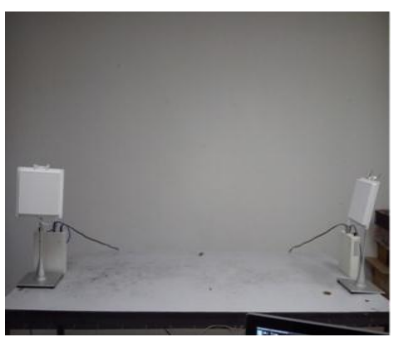

c) Rx facing out



b) Tx facing out

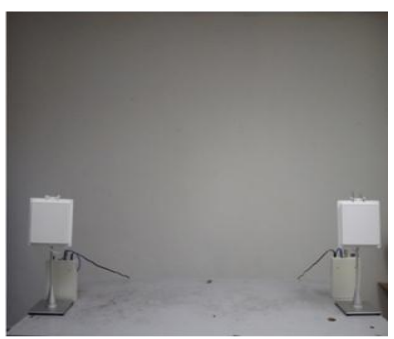

d) Tx-Rx facing out

Figure 5. Orientations of patch bi-directional antenna

\section{RESULTS AND ANALYSIS}

This section presents the findings on the effect of antenna orientation of monopole omnidirectional antenna at $2.4 \mathrm{GHz}$ and patch bi-directional antenna at $2.6 \mathrm{GHz}$ operations. The graphs were obtained by varying the gain at receiver from 0 to $27 \mathrm{~dB}$ for a fix distance between transmitter and receiver. 2500 packets have been transmitted, which consists of OFDM symbols of 1Megabytes of random number. The throughput is the percentage of packets successfully received by the receiver, while error rate is percentage of error out of the total packets received.

\subsection{Omni-directional monopole antenna at $2.4 \mathrm{GHz}$}

Figure 6 represents how much is the throughput received by receiver when increasing the gain at the receiver. Itcan be observed the ' $\mathrm{H}-\mathrm{H}$ ' orientation has gained $22 \%$ of throughput at $0 \mathrm{~dB}$ and increased to over $95 \%$ after $5 \mathrm{~dB}$ of $\mathrm{Rx}$ gain. On the other hand, the other orientations show no gain in throughput at $0 \mathrm{~dB}$, and only begin to gain an increase in throughput after $5 \mathrm{~dB}$. The ' $\mathrm{V}-\mathrm{V}$ ' orientation reaches more than $90 \%$ of throughput after $15 \mathrm{~dB}$ while both ' $\mathrm{V}-\mathrm{H}$ ' and ' $\mathrm{H}-\mathrm{V}$ ' only reach that point after $20 \mathrm{~dB}$. All the orientations close to $100 \%$ of throughput at $\mathrm{Rx}$ gain beyond $22 \mathrm{~dB}$. From the observations, antennas with same orientations ( $\mathrm{H}-\mathrm{H}$ and $\mathrm{V}-\mathrm{V}$ ) show better throughput performance than antennas with different orientations with ' $\mathrm{H}-\mathrm{H}$ ' orientation shows the best throughput performance, followed by ' $\mathrm{V}-\mathrm{V}$ ', ' $\mathrm{V}-\mathrm{H}$ ', and ' $\mathrm{H}-\mathrm{V}$ '. It is because antennas of $\mathrm{H}-\mathrm{H}$ ad V-V have same polarization (co-polar) that makes the antenna receiver receives throughput faster compared to cross-polar $(\mathrm{V}-\mathrm{H})$ and $(\mathrm{H}-\mathrm{V}) . \mathrm{H}-\mathrm{H}$ orientation (horizontal polarization) is better than $\mathrm{V}-\mathrm{V}$ because the wave propagates does not interact with ground plane and ceiling of the building which will add to the path loss to the V-V signal.

Subsequently, the antennas with same orientations also show better error rate performance than antennas with different orientations as presented in figure 7. From the figure, ' $\mathrm{H}-\mathrm{H}$ ' orientation shows much better performance than the other antenna orientations. It started showing reduction of error rate at $0 \mathrm{~dB}$ and approaching the minimum packet error rate of $10 \%$ after $10 \mathrm{~dB}$ while others only shown error reduction after $15 \mathrm{~dB}$ and approaching the same minimum error after $20 \mathrm{~dB}$ of Rx gain. In addition, although ' $\mathrm{V}-\mathrm{H}$ ' orientation shows slightly better performance in throughput than it counterparts ' $\mathrm{H}-\mathrm{V}$ ', it shows the worst performance in terms of error rate by around $40 \%$ higher than minimum error achieved by others after an $\mathrm{Rx}$ gain $25 \mathrm{~dB}$. 


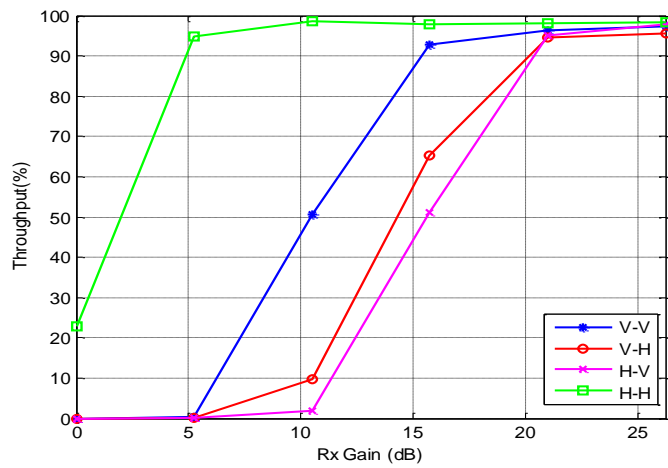

Figure 6. Throughput of different orientations of monopole omnidirectional antenna

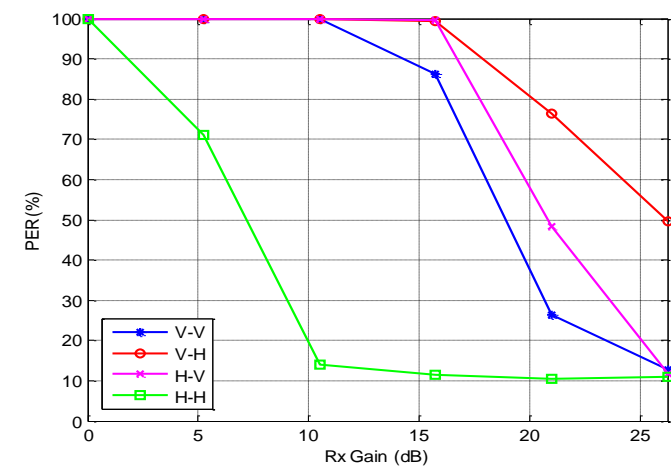

Figure 7. Packet error rate of different orientations of monopole omnidirectional antenna

\subsection{Bi-directional patch antenna at $2.6 \mathrm{GHz}$}

Unlike the omnidirectional antennas that been presented earlier, experimenting different orientations of bi-directional antenna on the system shows no effect on the system performance, neither in terms of throughput nor packet error rate as shown in Figure 8 and Figure 9 respectively.

In Figure 8, the throughput is constantly close to $100 \%$ across the Rx gain, regardless of the antenna orientations. Reciprocally, Figure 9 shows although the 'out-out' orientation shows decrement of error until $10 \mathrm{~dB}$, the error rate performance has saturated to about $10 \%$, which has been obtained consistently by other orientations throughout the Rx gain. While the performance is far from the anticipated, it might be due to the effect of saturation region of the patch antenna as shown in Figure 11.



Figure 8. Throughput of different orientations of patch bi-directional antenna

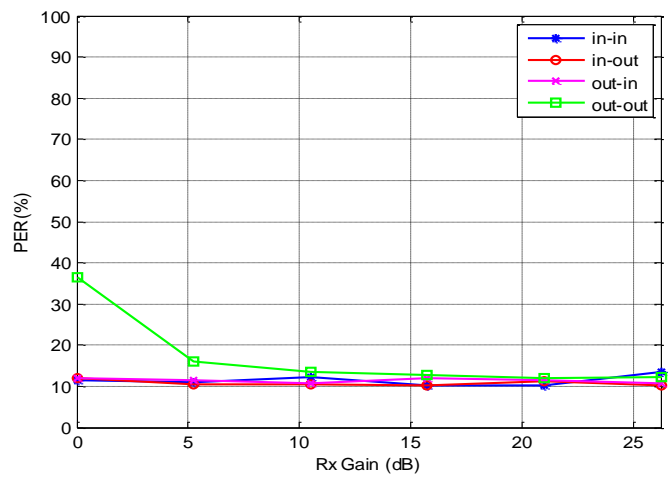

Figure 9. Packet error rate of different orientations of patch bi-directionsal antenna

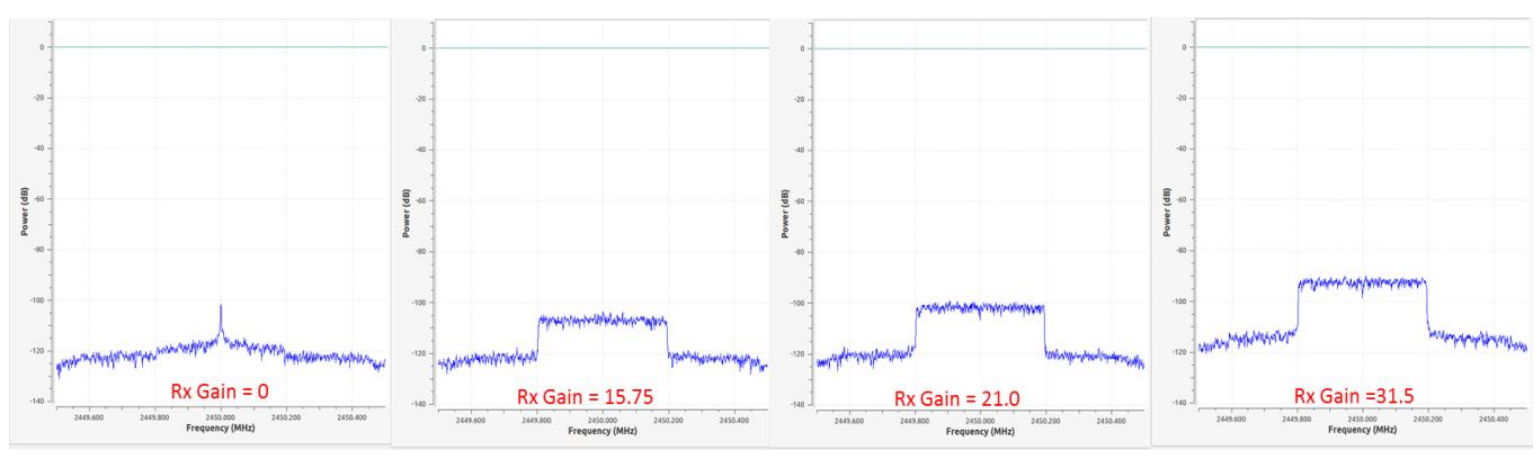

Figure 10. Received OFDM signal by monopole ominidirectional antenna with the corresponding Rx gain 


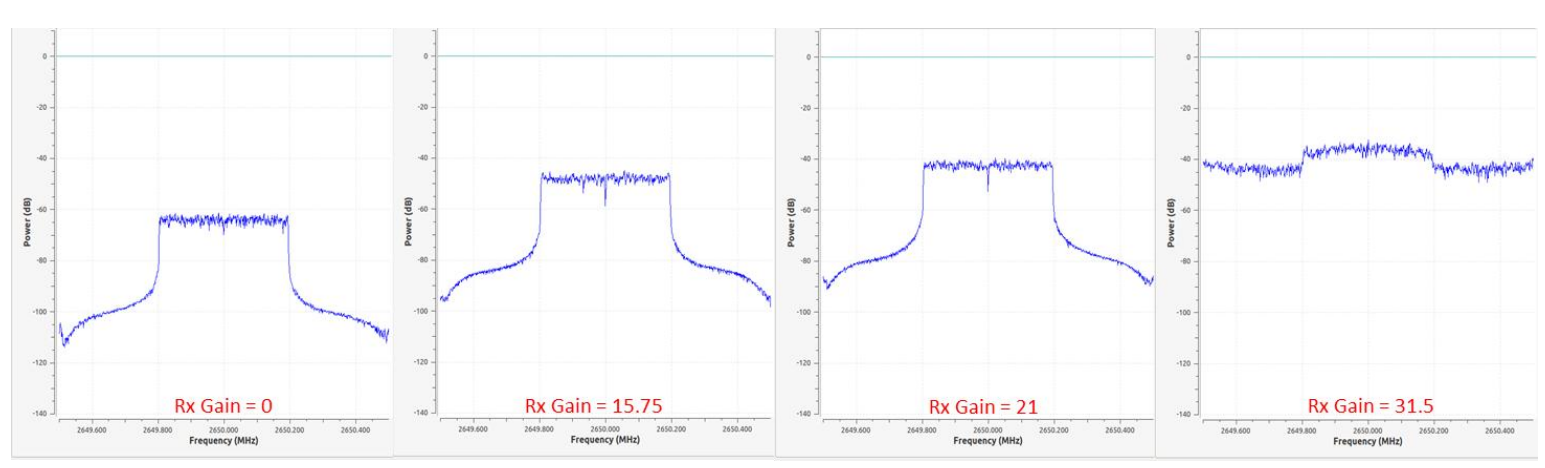

Figure 11. Received OFDM signal by patch bi-directional antenna with the corresponding Rx gain

From the observations on measured S11 and performance findings, it can be stated that the bidirectional antenna used in this study has better sensitivity and very high gain than the omnidirectional antenna. However, increasing the RX gain of the bi-directional antenna beyond $15 \mathrm{~dB}$ caused the signal to exceed the saturation point which caused the nonlinear signal in spite of power amplification. As such, the shape of OFDM is disappearing with the increased of RX gain as shown in Figure 11. Conversely, although omnidirectional shows poor S11, the reflectance from surroundings has helped in improving the performance as the Rx gain increased which is shown by better spectrum as shown in Figure 10.

\section{CONCLUSION}

From the exploration, it can be assured that SDR platform using USRP and GNU Radio can be used in prototyping the communication system. Careful precautions should be taken in determining proper configurations in order to avoid the hardware from operating at saturation region which caused non-linear system response. For this system, the nonlinear response caused the system to give peculiar signal output.

In demonstrating the importance of proper antenna orientation in wireless system, the findings show the omnidirectional antenna gives best performance when horizontally aligned in parallel orientation. However, the very high gain signal emitted from bi-directional antenna at $2.6 \mathrm{GHz}$ operation caused the signal to be saturated. Thus, the effect of this antenna orientation could not be observed.

\section{REFERENCES}

[1] T. H. T. Hwang, C. Y. C. Yang, G. W. G. Wu, S. L. S. Li, and G. Y. Li, "OFDM and Its Wireless Applications: A Survey," IEEE Trans. Veh. Technol, vol. 58, 2009.

[2] A. Al-Habashna, O. A. Dobre, R. Venkatesan, and D. C. Popescu, "Second-Order Cyclostationarity of Mobile WiMAX and LTE OFDM Signals and Application to Spectrum Awareness in Cognitive Radio Systems," IEEE J. Sel. Top. Signal Process., vol. 6, no. 1, pp. 26-42, Feb. 2012.

[3] X. Li, J. Vucic, V. Jungnickel, and J. Armstrong, "On the Capacity of Intensity-Modulated Direct-Detection Systems and the Information Rate of ACO-OFDM for Indoor Optical Wireless Applications," IEEE Trans. Commun., vol. 60, no. 3, pp. 799-809, Mar. 2012.

[4] N. Dupuis, C. R. Doerr, L. Zhang, L. Chen, N. J. Sauer, P. Dong, L. L. Buhl, and D. Ahn, "InP-Based Comb Generator for Optical OFDM," J. Light. Technol, vol. 30, no. 4, pp. 466-472, Feb 2012.

[5] J. L. Wei, J. D. Ingham, D. G. Cunningham, R. V. Penty, and I. H. White, "Performance and Power Dissipation Comparisons Between $28 \mathrm{~Gb} / \mathrm{s}$ NRZ, PAM, CAP and Optical OFDM Systems for Data Communication Applications," J. Light. Technol, vol. 30, no. 20, pp. 3273-3280, Oct 2012.

[6] S. Sun, Y. Ju, and Y. Yamao, "Overlay cognitive radio OFDM system for 4G cellular networks," IEEE Wirel. Commun., vol. 20, no. 2, pp. 68-73, Apr 2013.

[7] B. Jameson, D. Garmatyuk, and Y. T. J. Morton, "Cognitive radar for indoor positioning with a software-defined UWB OFDM system,” in 2012 IEEE Radar Conference, 2012, pp. 0465-0470.

[8] S. Sen, "OFDM Radar Space-Time Adaptive Processing by Exploiting Spatio-Temporal Sparsity," IEEE Trans. Signal Process., vol. 61, no. 1, pp. 118-130, Jan. 2013.

[9] T. Liu, N. Cao, M. Mao, F. Wang, and Y. Du, "Waveform design and optimization for OFDM radar signal based on fractional Fourier transform," in The 2014 2nd International Conference on Systems and Informatics (ICSAI 2014), 2014, pp. 724-729.

[10] S. Shearman and J. Kimery, "Software Defined Radio Prototyping Platforms Enable a Flexible Approach to Design," Microw. Mag. IEEE, 2012.

[11] J. F. Troncoso, "Software Defined Radio." 
[12] S. Semenov and E. Krouk, "Modulation and coding techniques in wireless communications." John Wiley \& Sons, 2011.

[13] K. Fazel and S. Kaiser, "Multi-Carrier and Spread Spectrum Systems: From OFDM and MC-CDMA to LTE and WiMAX: Second Edition.” 2008, pp. 1-360.

[14] F. M. Andreas, Wireless communications, 2nd ed. John Wiley \& Sons, 2011.

[15] “GNU Radio Manual and C++ API Reference: Main Page.” [Online]. Available: http://gnuradio.org/doc/doxygen/.

[16] J. G. Proakis and M. Salehi, Digital Communications. 2008, p. 1150. 